\title{
Random subgraphs make identification affordable
}

\author{
Florent Foucaud, Guillem Perarnau and Oriol Serra
}

October 8, 2018

\begin{abstract}
An identifying code of a graph is a dominating set which uniquely determines all the vertices by their neighborhood within the code. Whereas graphs with large minimum degree have small domination number, this is not the case for the identifying code number (the size of a smallest identifying code), which indeed is not even a monotone parameter with respect to graph inclusion.

We show that every graph $G$ with $n$ vertices, maximum degree $\Delta=\omega(1)$ and minimum degree $\delta \geq c \log \Delta$, for some constant $c>0$, contains a large spanning subgraph which admits an identifying code with size $O\left(\frac{n \log \Delta}{\delta}\right)$. In particular, if $\delta=\Theta(n)$, then $G$ has a dense spanning subgraph with identifying $\operatorname{code} O(\log n)$, namely, of asymptotically optimal size. The subgraph we build is created using a probabilistic approach, and we use an interplay of various random methods to analyze it. Moreover we show that the result is essentially best possible, both in terms of the number of deleted edges and the size of the identifying code.
\end{abstract}

\section{Introduction}

Consider any graph parameter that is not monotone with respect to graph inclusion. Given a graph $G$, a natural problem in this context is to study the minimum value of this parameter over all spanning subgraphs of $G$. In particular, how many edge deletions are sufficient in order to obtain from $G$ a graph with near-optimal value of the parameter? Herein, we use random methods to study this question with respect to the identifying code number of a graph, a well-studied non-monotone parameter. An identifying code of graph $G$ is a set $C$ of vertices which is a dominating set, and such that the closed neighborhood within $C$ of each vertex $v$ uniquely determines $v$.

Identifying codes were introduced in 1998 in [19] and have been studied extensively in the literature since then. We refer to 24] for an on-line bibliography. One of the interests of this notion lies in their applications to the location of threats in facilities [28] and error-detection in computer networks [19]. One can also mention applications to routing [23], to bio-informatics [18] and to measuring the first-order logical complexity of graphs [20]. Let us also mention that identifying codes are special cases of the more general notion of test covers of hypergraphs, see e.g. [11, 26] (test covers are also the implicit object of Bondy's celebrated theorem on induced subsets [7]).

Let $G$ be a simple, undirected and finite graph. The open neighborhood of a vertex $v$ in $G$ is the set of vertices in $V(G)$ that are adjacent to it, and will be denoted $N_{G}(v)$. The closed neighborhood of a vertex $v$ in $G$ is defined as $N_{G}[v]=N_{G}(v) \cup\{v\}$. The degree of a vertex $u \in V(G)$, is defined as $d(v)=\left|N_{G}(v)\right|$. Similarly, we define, for a set $S \subseteq V(G), N_{G}(S)=\bigcup_{v \in S} N_{G}(v)$ and $N_{G}[S]=\bigcup_{v \in S} N_{G}[v]$. If two distinct vertices $u, v$ are such that $N[u]=N[v]$, they are called twins. The symmetric difference between two sets $A$ and $B$ is denoted by $A \oplus B$. 
Given a graph $G$ and a subset $C$ of vertices of $G, C$ is called a dominating set if each vertex of $V(G) \backslash C$ has at least one neighbor in $C$. The set $C$ is called a separating set of $G$ if for each pair $u, v$ of vertices of $G, N[u] \cap C \neq N[v] \cap C$ (equivalently, $(N[u] \oplus N[v]) \cap C \neq \emptyset$ ). If $x \in N[u]$, we say that $x$ dominates $u$. If $x \in N[u] \oplus N[v]$, we say that $x$ separates $u, v$.

Definition 1. A subset of vertices of a graph $G$ which is both a dominating set and a separating set is called an identifying code of $G$.

The following observation gives an equivalent condition for a set to be an identifying code, and follows from the fact that for two vertices $u, v$ at distance at least 3 from each other, $N[u] \oplus N[v]=N[u] \cup N[v]$.

Observation 2. For a graph $G$ and a set $C \subseteq V(G)$, if $C$ is dominating and $N[u] \cap C \neq N[v] \cap C$ for each pair of vertices $u, v$ at distance at most two from each other, then $C$ is an identifying code of the graph.

The minimum size of a dominating set of graph $G$, its domination number, is denoted by $\gamma(G)$. Similarly, the minimum size of an identifying code of $G, \gamma^{\mathrm{ID}}(G)$, is the identifying code number of $G$. It is known that for any twin-free graph $G$ on $n$ vertices having at least one edge, we have:

$$
\left\lceil\log _{2}(n+1)\right\rceil \leq \gamma^{\mathrm{ID}}(G) \leq n-1
$$

The lower bound was proved in [19] and the upper bound, in [6, 17. Both bounds are tight and all graphs reaching these two bounds have been classified (see [25, for the lower bound and [12] for the upper bound). Other papers studying bounds and extremal graphs for identifying codes are e.g. [9, 13, 14].

In view of the above lower bound, we say that an identifying code $C$ of $G$ is asymptotically optimal if

$$
|C|=O(\log n)
$$

The problem we will address in this paper is to deal with graphs that have a large identifying code number, or are not even identifiable. Our approach will consist in slightly modifying such a graph in order to decrease its identifying code number and obtain an asymptotically optimal identifying code, unless its domination number prevents us from doing so.

One of the reasons for a graph to have a large identifying code number is that it has a large domination number (this one being a monotone parameter under edge deletion). For instance, we need roughly $n / 3$ vertices to dominate all the vertices in a path of order $n$. When this is the case, we cannot expect to decrease much the size of a minimum identifying code by deleting edges from $G$, as the deletion of edges cannot decrease the domination number.

However, there are many graphs with small domination number where the identifying code number is very large [12, 14]. Typically, this phenomenon appears in graphs having a specific, "rigid", structure. Supporting this intuition, Frieze, Martin, Moncel, Ruszinkó and Smyth [16] have shown that the random graph $G(n, p)$ with $p \in(0,1)$, admits an asymptotically optimal identifying code. In particular, they prove in [16] that

$$
\gamma^{\mathrm{ID}}(G(n, p))=(1+o(1)) \frac{2 \log n}{\log (1 / q)},
$$

where $q=p^{2}+(1-p)^{2}$. This suggests that the lack of structure in dense graphs implies the existence of a small identifying code. 
Our results and structure of the paper. In Section 2, we prove our main result by selecting at random a small set of edges that can be deleted to "add some randomness" in the graph,

Theorem 3. For any graph $G$ on $n$ vertices ( $n$ large enough) with maximum degree $\Delta=\omega(1)$ and minimum degree $\delta \geq 66 \log \Delta$, there exists a subset of edges $F \subset E(G)$ of size

$$
|F| \leq 83 n \log \Delta,
$$

such that

$$
\gamma^{I D}(G \backslash F) \leq 134 \frac{n \log \Delta}{\delta} .
$$

Observe that when $\delta=\Theta(n)$, this result is similar to the one in [16].

We then show in Section 3 that our result is asymptotically best possible in terms of both the number of deleted edges and of the size of the final identifying code for any graph with $\Delta=\operatorname{Poly}(\delta)$. For smaller values of the minimum degree, we prove that our result is almost optimal. We also show that the two assumptions $\Delta=\omega(1)$ and $\delta \geq c \log \Delta$ for some constant $c$ are necessary.

We present some consequences of our result in Section 4 When considering the case of adding edges to the graph, we get analogous (symmetric) results, showing that every graph is a large spanning subgraph of some graph that admits a small identifying code. This result also turns out to be tight. We also describe an application to the closely related topic of watching systems.

The paper concludes with some final remarks and open problems.

Our methods. To show our results, we use the technique of defining a suitable random spanning subgraph of $G$ : we first randomly choose a code $C$, and then we randomly delete edges among the edges containing vertices of $C$. We then analyze the construction by applying concentration inequalities and the use of the local lemma.

A similar approach has been used in the literature when considering random subgraphs of a graph: for any graph $G$, consider the graph $G_{p}$ to be the subgraph of $G$ obtained by keeping each edge from $E(G)$ independently with probability $p$. The behavior of random subgraphs of graphs $G$ with minimum degree $\delta$, inspired by applications in epidemiology or social networks, has been widely studied [10, 15, 21, 22. A well-known instance of this problem is the classical Erdös-Rényi random graph $G(n, p)$ where $G=K_{n}$, the complete graph of order $n$. In most of the cases, it was shown that many similarities exist between $G_{p}$ and the random graph $G(\delta, p)$. The connectivity of a random subgraph of a graph, where every edge has a different probability of being deleted, has been studied in [1]. Our random subgraph model is adapted to the analysis of identifying codes, and can be seen as a weighted version of $G_{p}$.

\section{Main theorem}

In this section, we prove Theorem 3, We will need some tools and lemmas.

\section{$2.1 \quad$ Important tools and lemmas}

In our proofs, we will repeatedly use the Chernoff inequality for the sum of independent bounded random variables: 
Lemma 4 (Chernoff inequality [2, Corollary A.1.14]). Let $X_{1}, \ldots, X_{N}$ be independent Bernoulli random variable with probability $p_{i}$ and define $X=\sum_{i=1}^{N} X_{i}$. Then, for all $\varepsilon>0$,

$$
\operatorname{Pr}(|X-\mathbb{E}(X)| \geq \varepsilon \mathbb{E}(X))<2 e^{-c_{\varepsilon} \mathbb{E}(X)},
$$

where

$$
c_{\varepsilon}=\min \left\{(1+\varepsilon) \log (1+\varepsilon)-\varepsilon, \frac{\varepsilon^{2}}{2}\right\} .
$$

In what follows, for any set of vertices $B \subseteq V(G)$ and any $v \in V(G)$, we let $N_{G}^{B}(v)=N_{G}(v) \cap B$ be the set of neighbors of $v$ in $B$. Analogously, $N_{G}^{B}[v]=N_{G}[v] \cap B$. We denote by $d_{B}(v)=\left|N_{G}^{B}(v)\right|$, the degree of $v$ within set $B$.

Definition 5. Given a graph $G$ and $B \subseteq V(G)$, a function $f: V(G) \rightarrow \mathbb{R}^{+} \cup\{0\}$ is said to be $(G, B)$-bounded if for each vertex $u, f(u) \leq d_{B}(u)$ and for each pair $u, v$ of vertices with $d_{B}(u) \geq d_{B}(v), f(u) / d_{B}(u) \leq f(v) / d_{B}(v)$. Given a $(G, B)$-bounded function $f$, we define the random spanning subgraph $G(B, f)$ of $G$ as follows:

- $G(B, f)$ contains all edges of the subgraph $G[V(G) \backslash B]$ induced by $V(G) \backslash B$, and

- each edge $u v$ incident with $B$ is independently chosen to be in $G(B, f)$ with probability $1-p_{u v}$, where

$$
p_{u v}=\frac{1}{4}\left(\frac{f(u)}{d_{B}(u)}+\frac{f(v)}{d_{B}(v)}\right) .
$$

Observe that, since $f(u) \leq d_{B}(u)$ for each vertex $u \in V(G)$, we have $p_{u v} \leq 1 / 2$.

The next lemma gives an exponential upper bound on the probability that two vertices of $G(B, f)$ are not separated by $B$. This lemma is a crucial one in our main proof.

Lemma 6. Let $G$ be a graph, $B \subseteq V(G)$, and $f$ a $(G, B)$-bounded function. In the random subgraph $G(B, f)$, for every pair $u, v$ of distinct vertices with $d_{B}(u) \geq d_{B}(v)$, we have

$$
\operatorname{Pr}\left(N_{G(B, f)}^{B}[u]=N_{G(B, f)}^{B}[v]\right) \leq e^{-3 f(u) / 16} .
$$

Proof. Consider the following partition of $S=N_{G}^{B}[u] \cup N_{G}^{B}[v]$ into three parts: $S_{1}$, the vertices of $B$ dominating $u$ but not $v ; S_{2}$, the vertices of $B$ dominating $v$ but not $u$; and $S_{3}$, the vertices of $B$ dominating both $u$ and $v$.

Let $D$ be the random variable which gives the size of the symmetric difference of $N_{G(B, f)}^{B}[u]$ and $N_{G(B, f)}^{B}[v]$. The statement of the lemma is equivalent to $\operatorname{Pr}(D=0)<e^{-3 f(u) / 16}$.

The random variable $D=\left|N_{G(B, f)}^{B}[u] \oplus N_{G(B, f)}^{B}[v]\right|$ can be written as the sum of independent Bernoulli variables

$$
D=\sum_{w \in S} D_{w}
$$

where $D_{w}=1$ if and only if $w$ dominates precisely one of the two vertices $u$ or $v$ in $G(B, f)$. Therefore, fro any $w \notin\{u, v\}$,

$$
\operatorname{Pr}\left(D_{w}=1\right)= \begin{cases}1-p_{u w} & w \in S_{1} \\ 1-p_{v w} & w \in S_{2} \\ p_{u w}\left(1-p_{v w}\right)+p_{v w}\left(1-p_{u w}\right) & w \in S_{3}\end{cases}
$$


Since we want to bound from above the probability that $D=0$, we can always assume that $u, v \notin N_{G(B, f)}^{B}[u] \oplus$ $N_{G(B, f)}^{B}[v]$. Recall that $d_{B}(u) \geq d_{B}(v)$. By the definition of a $(G, B)$-bounded function, we have that $p_{u w} \leq p_{v w}$ for each $w \in S_{3}$. Since $x(1-x)$ has a unique maximum at $x=1 / 2$ and $p_{u w}, p_{v w} \leq 1 / 2$, we also have:

$$
p_{v w}\left(1-p_{u w}\right) \geq p_{u w}\left(1-p_{u w}\right) \geq \frac{f(u)}{4 d_{B}(u)}\left(1-\frac{f(u)}{4 d_{B}(u)}\right)=g(u),
$$

for each $w \in S_{3}$.

For $w \in S$, denote by $q_{w}$ the parameter of the Bernoulli random variable $D_{w}$. Then,

$$
\begin{aligned}
\mathbb{E}(D) & \geq \sum_{w \in N_{G}^{B}(u)} q_{w} \\
& =\sum_{w \in S_{1}} q_{w}+\sum_{w \in S_{3}} q_{w} \\
& =\sum_{w \in S_{1}}\left(1-p_{u w}\right)+\sum_{w \in S_{3}}\left(p_{u w}\left(1-p_{v w}\right)+p_{v w}\left(1-p_{u w}\right)\right) \\
& \geq \sum_{w \in S_{1}} p_{u w}\left(1-p_{u w}\right)+\sum_{w \in S_{3}} p_{u w}\left(1-p_{u w}\right) \\
& \geq g(u) d_{B}(u) \\
& =\frac{f(u)}{4}\left(1-\frac{f(u)}{4 d_{B}(u)}\right) \\
& \geq \frac{3}{16} f(u)
\end{aligned}
$$

Finally, we have that

$$
\operatorname{Pr}(D=0)=\prod_{w \in S}\left(1-q_{w}\right) \leq e^{-\sum_{w \in S} q_{w}}=e^{-\mathbb{E}(D)} \leq e^{-3 f(u) / 16},
$$

and the lemma follows.

In the proof of our main result, we will use the following version of the Lovász local lemma, which can be found in e.g. 2, Corollary 5.1.2] (the lower bound on $\operatorname{Pr}\left(\bigcap_{i=1}^{M} \overline{E_{i}}\right)$ can be derived from the general local lemma, see [2, Lemma 5.1.1], by setting $\left.x_{i}=e \cdot p_{L L}\right)$.

Lemma 7 (Symmetric Local Lemma). Let $\mathcal{E}=\left\{E_{1}, \ldots, E_{M}\right\}$ be a set of (typically "bad") events such that each $E_{i}$ is mutually independent of $\mathcal{E} \backslash\left(\mathcal{D}_{i} \cup\left\{E_{i}\right\}\right)$ for some $\mathcal{D}_{i} \subseteq \mathcal{E}$. Let $d_{L L}=\left|\mathcal{D}_{i}\right|$, and suppose that there exists a real $0<p_{L L}<1$ such that, for each $1 \leq i \leq M$,

- $\operatorname{Pr}\left(E_{i}\right) \leq p_{L L}$, and

- $e \cdot p_{L L} \cdot\left(d_{L L}+1\right) \leq 1$.

Then $\operatorname{Pr}\left(\bigcap_{i=1}^{M} \overline{E_{i}}\right) \geq\left(1-e \cdot p_{L L}\right)^{M}>0$.

\subsection{Proof of the main result}

We are now ready to prove the main theorem. 
Proof of Theorem 3. The proof is structured in the following steps:

1. We select a set $C$ at random, where each vertex is selected independently with probability $p$. Using the Chernoff inequality, we estimate the probability of the event $A_{C}$ that $C$ is small enough for our purposes. From $C$, we construct the spanning subgraph $G(C, f)$ of $G$ as given in Definition [5, for some suitable function $f$.

2. We use the local lemma (Lemma 77) and Lemma 6 to bound from below the probability that the following events (whose conjunction we call $A_{L L}$ ) hold jointly: (i) in $G(C, f)$, each pair of vertices that are at distance at most 2 from each other are separated by $C$; and (ii) for each such pair and each member of this pair in $G$, its degree within $C$ in $G$ is close to its expected value $d(v) p$. We show that with nonzero probability, $A_{C}$ and $A_{L L}$ hold jointly.

3. We find a dominating set $D$ of $G$ with $|D|=O(|C|)$; by Observation 2 if $A_{L L}$ holds, then $C \cup D$ is an identifying code.

4. Finally, we show that, subject to $A_{C}$ and $A_{L L}$, the expected number of deleted edges is as small as desired.

\section{Step 1. Constructing $C$ and $G(C, f)$}

Let $C \subseteq V(G)$ be a subset of vertices, where each vertex $v$ in $G$ is chosen to be in $C$ independently with probability

$$
p=\frac{66 \log \Delta}{\delta} .
$$

Observe that $p \leq 1$ since $\delta \geq 66 \log \Delta$.

Consider the random variable $|C|$ and recall that $\mathbb{E}(|C|)=n p$.

Define $A_{C}$ to be the event that

$$
|C| \leq 2 n p=\frac{132 n \log \Delta}{\delta}
$$

Since the choices of the elements in $C$ are done independently, by setting $\varepsilon=1$ in Lemma 4 notice that $c_{\varepsilon}>1 / 3$, we have

$$
\operatorname{Pr}\left(\overline{A_{C}}\right)<e^{-\frac{22 n \log \Delta}{\delta}}
$$

We let

$$
f(u)=\min \left(66 \log \Delta, d_{C}(u)\right) .
$$

Observe that $f$ is $(G, C)$-bounded. We construct $G(C, f)$ as the random spanning subgraph of $G$ given in Definition [5, where each edge $u v$ incident to a vertex of $C$ is deleted with probability $p_{u v}$.

\section{Step 2. Applying the local lemma}

Let $u, v$ be a pair of vertices at distance at most 2 in $G$. We define the following events:

- $A_{u v}$ is the event that there exists a vertex $w \in\{u, v\}$ such that the degree of $w$ within $C$ is deviating from its expected value $d(w) p$ by half, i.e. $\left|d_{C}(w)-d(w) p\right| \geq \frac{d(w) p}{2}$;

- $B_{u v}$ is the event that $N_{G(C, f)}^{C}[u]=N_{G(C, f)}^{C}[v]$; 
- $E_{u v}$ is the event that $A_{u v}$ or $B_{u v}$ occurs;

- $A_{L L}$ is the event that no event $E_{u v}$ occurs.

In order to apply the Local Lemma, we wish to upper bound the probability of $E_{u v}$. We have:

$$
\begin{aligned}
\operatorname{Pr}\left(E_{u v}\right) & \leq \operatorname{Pr}\left(A_{u v}\right)+\operatorname{Pr}\left(B_{u v}\right) \\
& =\operatorname{Pr}\left(A_{u v}\right)+\operatorname{Pr}\left(B_{u v} \mid A_{u v}\right) \cdot \operatorname{Pr}\left(A_{u v}\right)+\operatorname{Pr}\left(B_{u v} \mid \overline{A_{u v}}\right) \cdot \operatorname{Pr}\left(\overline{A_{u v}}\right) .
\end{aligned}
$$

Let us upper bound $\operatorname{Pr}\left(A_{u v}\right)$. We use Lemma 4 with $\varepsilon=1 / 2$. Observe that $c_{\varepsilon}>\frac{1}{10}$, and thus

$$
\begin{aligned}
\operatorname{Pr}\left(A_{u v}\right) & <\operatorname{Pr}\left(\left|d_{C}(u)-d(u) p\right| \geq \frac{d(u) p}{2}\right)+\operatorname{Pr}\left(\left|d_{C}(v)-d(v) p\right| \geq \frac{d(v) p}{2}\right) \\
& \leq 2 e^{-\frac{1}{10} d(u) p}+2 e^{-\frac{1}{10} d(v) p} \\
& =2 e^{-\frac{66 d(u) \log \Delta}{100}}+2 e^{-\frac{66 d(v) \log \Delta}{100}} \\
& \leq 4 e^{-\frac{33 \log \Delta}{5}} \\
& \leq 4 \Delta^{-\frac{33}{5}}
\end{aligned}
$$

Next, we give an upper bound for $\operatorname{Pr}\left(B_{u v} \mid \overline{A_{u v}}\right)$. For such a purpose, we apply Lemma 6 with $B=C$ and $f(u)=\min \left(66 \log \Delta, d_{C}(u)\right)$. Observe that $f$ is $(G, C)$-bounded. Since $A_{u v}$ does not hold, we know that $d_{C}(u)$ and $d_{C}(v)$ are large enough, i.e. for $w \in\{u, v\}, d_{C}(w) \geq \frac{d(w) p}{2} \geq \frac{\delta p}{2}=33 \log \Delta$; thus $f(u), f(v) \geq 33 \log \Delta$. We have:

$$
\operatorname{Pr}\left(B_{u v} \mid \overline{A_{u v}}\right) \leq e^{-\frac{3 \cdot 33 \log \Delta}{16}} \leq \Delta^{-\frac{99}{16}}
$$

The probability that the event $E_{u v}$ holds is

$$
\begin{aligned}
\operatorname{Pr}\left(E_{u v}\right) \leq & \operatorname{Pr}\left(A_{u v}\right)+\operatorname{Pr}\left(B_{u v} \mid A_{u v}\right) \cdot \operatorname{Pr}\left(A_{u v}\right)+\operatorname{Pr}\left(B_{u v} \mid \overline{A_{u v}}\right) \cdot \operatorname{Pr}\left(\overline{A_{u v}}\right) \\
& \leq 4 \Delta^{-\frac{33}{5}}+1 \cdot 4 \Delta^{-\frac{33}{5}}+\Delta^{-\frac{99}{16}} \cdot 1 \\
& \leq 2 \Delta^{-\frac{99}{16}}=p_{L L}
\end{aligned}
$$

where we used $\Delta=\omega(1)$.

We now note that each event $E_{u v}$ is mutually independent of all but at most $2 \Delta^{6}$ events $E_{u^{\prime} v^{\prime}}$. Indeed, $E_{u v}$ depends on the random variables determining the existence of the edges incident to $u$ and $v$. This is given by probabilities $p_{u w}$ and $p_{v w}$ that depend on $d_{C}(w)$, where $w$ is at distance at most one from either $u$ or $v$. Thus, $E_{u v}$ depends only on the vertices at distance at most two from either $u$ or $v$ belonging to $C$. In other words, $E_{u v}$ and $E_{u^{\prime} v^{\prime}}$ are mutually independent unless there exist a vertex $w$ at distance at most two from both pairs; in other words, $d\left(\{u, v\},\left\{u^{\prime}, v^{\prime}\right\}\right) \leq 4$. Hence, there are at most $2 \Delta^{4}$ choices for the vertex among $\left\{u^{\prime}, v^{\prime}\right\}$ that is closest from $\{u, v\}$ (say $u^{\prime}$ ), and at most $\Delta^{2}$ additional choices for $v^{\prime}$, since $d\left(u^{\prime}, v^{\prime}\right) \leq 2$.

Therefore, we can apply Lemma 7 if

$$
e \cdot 2 \Delta^{-\frac{99}{16}} \cdot\left(2 \Delta^{6}+1\right) \leq 1
$$

which holds since $\Delta=\omega(1)$.

Now, by Lemma 7 and since there are at most $\frac{n \Delta^{2}}{2}$ events $E_{u v}$ (one for each pair of vertices at distance at 
most 2 from each other) and $p_{L L}=2 \Delta^{-\frac{99}{16}}$

$$
\operatorname{Pr}\left(A_{L L}\right) \geq\left(1-e \cdot p_{L L}\right)^{M} \geq e^{-2 e \cdot p_{L L} M} \geq e^{-2 e n \Delta^{2-\frac{99}{16}}},
$$

where we have used $(1-x)=e^{-x(1-O(x))} \geq e^{-2 x}$, if $x=o(1)$.

\section{Step 3. Revealing the identifying code}

Let us lower bound the probability that both $A_{C}$ and $A_{L L}$ hold, by using Inequalities 3 and 5 .

$$
\begin{aligned}
\operatorname{Pr}\left(A_{C} \cap A_{L L}\right) & \geq \operatorname{Pr}\left(A_{L L}\right)-\operatorname{Pr}\left(\overline{A_{C}}\right) \\
& \geq e^{-2 e n \Delta^{2-\frac{99}{16}}}-e^{-\frac{22 n \log \Delta}{\delta}},
\end{aligned}
$$

which is strictly positive if

$$
\frac{22 \log \Delta}{\delta}>2 e \Delta^{2-\frac{99}{16}}
$$

which holds since $n$ is large (and hence $\Delta=\omega(1)$ is large too), and $\delta \leq \Delta$.

Hence, there exists a set $C$ of size $132 \frac{n \log \Delta}{\delta}$ such that all vertices at distance 2 from each other are separated by $C$, and such that the degree in $C$ of all vertices is large.

In order to build an identifying code, we must also make sure that all vertices are dominated. It is well-known that for any graph $G, \gamma(G) \leq(1+o(1)) \frac{n \log \delta}{\delta}$ (see e.g. [2, Theorem 1.2.2]). Hence, we select a dominating set $D$ of $G$ with size $(1+o(1)) \frac{n \log \delta}{\delta}$. Then, by Observation 2, $C \cup D$ is an identifying code of size at most

$$
(132+1+o(1)) \frac{n \log \Delta}{\delta} \leq 134 \frac{n \log \Delta}{\delta}
$$

\section{Step 4. Estimating the number of deleted edges}

Let $Y=|E(G) \backslash E(G(C, f))|$ be the number of edges we have deleted from $G$ to obtain $G(C, f)$. Recall that each edge $u v \in E(G)$ is deleted independently from $G$ with probability

$$
p_{u v}=\frac{1}{4}\left(\frac{f(u)}{d_{C}(u)}+\frac{f(v)}{d_{C}(v)}\right),
$$

if one of its endpoints is in $C$.

Since $\operatorname{Pr}\left(A_{C} \cap A_{L L}\right)>0$, there is a small identifying code of $G$ obtained by deleting at most $\mathbb{E}\left(Y \mid A_{C} \cap A_{L L}\right)$ edges. We next give an upper bound for $\mathbb{E}\left(Y \mid A_{C} \cap A_{L L}\right)$. If both $A_{C}$ and $A_{L L}$ hold, then

$$
p_{u v} \leq \frac{1}{4}\left(\frac{66 \log \Delta}{d_{C}(u)}+\frac{66 \log \Delta}{d_{C}(v)}\right)
$$

The expected number of deleted edges is

$$
\mathbb{E}\left(Y \mid A_{C} \cap A_{L L}\right)=\sum_{\substack{u v \in E(G) \\(\{u, v\} \cap C) \neq \emptyset}} p_{u v}
$$

Observe that in order to estimate this quantity, we can split the two additive terms in each $p_{u v}$ : for every $u \notin C$, we sum all the terms $\frac{66 \log \Delta}{4 d_{C}(u)}$ for all $v \in C$ being neighbors of $u$; for every $u \in C$, we sum all the terms $\frac{66 \log \Delta}{4 d_{C}(u)}$ 
for all $v \in V(G)$ being neighbors of $u$.

$$
\begin{aligned}
\mathbb{E}\left(Y \mid A_{C} \cap A_{L L}\right) & \leq \frac{1}{4}\left(\sum_{u \notin C} \sum_{v \in N_{G}^{C}(u)} \frac{66 \log \Delta}{d_{C}(u)}+\sum_{u \in C} \sum_{v \in N_{G}(u)} \frac{66 \log \Delta}{d_{C}(u)}\right) \\
& \leq \frac{1}{4}\left(\sum_{u \notin C} d_{C}(u) \frac{66 \log \Delta}{d_{C}(u)}+\sum_{u \in C} d(u) \frac{66 \log \Delta}{d_{C}(u)}\right) \\
& \leq \frac{1}{4}\left(|V(G) \backslash C| \cdot 66 \log \Delta+\sum_{u \in C} 2 \frac{66 \log \Delta}{p}\right) \\
& \leq \frac{1}{4}(n \cdot 66 \log \Delta+2|C| \delta) \\
& \leq \frac{66 n \log \Delta+264 n \log \Delta}{4} \\
& \leq 83 n \log \Delta
\end{aligned}
$$

where we used the fact (implied by $A_{L L}$ ) that for any vertex $v, \frac{d(v) p}{2} \leq d_{C}(v)$ at the second line, and that $A_{C}$ implies $|C| \leq 132 \frac{n \log \Delta}{\delta}$ at the fifth line.

Summarizing, we have shown the existence of a small identifying code in a spanning subgraph of $G$ obtained by deleting at most $\mathbb{E}\left(Y \mid A_{C} \cap A_{L L}\right)$ edges from $G$, which completes the proof.

\section{Asymptotic optimality of Theorem 3}

In this section, we discuss the optimality of Theorem 3 first with respect to the size of the constructed code and the number of deleted edges, and then with respect to the hypothesis $\Delta=\omega(1)$ and $\delta \geq 66 \log \Delta$.

\subsection{On the size of the code and the number of deleted edges}

Charon, Honkala, Hudry and Lobstein showed that deleting an edge from $G$ can decrease by at most 2 the identifying code number of a graph $[8$. That is, for any graph $G$ and any edge $u v \in E(G)$,

$$
\gamma^{\mathrm{ID}}(G) \leq \gamma^{\mathrm{ID}}(G \backslash u v)+2 .
$$

This directly implies that for every graph with linear identifying code number, one needs to delete a subset $F$ of at least $\Omega(n)$ edges, to get a graph with $\gamma^{\mathrm{ID}}(G \backslash F)=o(n)$.

We will show that, indeed, one needs to delete at least $\Omega(n \log n)$ edges from the complete graph to get a graph with an asymptotically optimal identifying code. Using this, we will derive a family of graphs with arbitrary minimum degree $\delta$, that asymptotically attains the bound of Theorem 3, both in number of edges and size of the minimum code, when $\Delta=\operatorname{Poly}(\delta)$.

First of all, we prove that every graph with an asymptotically optimal identifying code cannot contain too few edges.

Lemma 8. For any $M^{\prime} \geq 0$, there exists a constant $c_{0}>0$ such that any graph $G$ with $\gamma^{I D}(G) \leq M^{\prime} \log n$ contains at least $c_{0} n \log n$ edges. 
Proof. Set $\alpha_{0}$ as the smallest positive root of

$$
f(\alpha)=\alpha \log \left(\frac{M^{\prime}+\alpha}{\alpha} e\right)-1 / 2 .
$$

Note that $f(\alpha)$ is well-defined since $\lim _{\alpha \rightarrow 0} f(\alpha)=-1 / 2$ and $f(1)=\log \left(M^{\prime}+1\right)+1 / 2>0$.

Suppose by contradiction that there exists a graph $G$ containing less than $c_{0} n \log n$ edges, with $c_{0}=\alpha_{0} / 4$, that admits an identifying code $C$ of size at most $M^{\prime} \log n$. Let $U$ be the subset of vertices of degree at least $\alpha_{0} \log n$. Notice that

$$
|U| \leq \frac{2|E(G)|}{\alpha_{0} \log n} \leq \frac{2 c_{0}}{\alpha_{0}} n=\frac{n}{2} .
$$

Since $|C| \leq M^{\prime} \log n$ and any $v \in V(G) \backslash U$ has degree smaller than $\alpha_{0} \log n$, the number of possible nonempty sets $N_{G}[v] \cap C$, is smaller than

$$
\begin{aligned}
\sum_{i=1}^{\alpha_{0} \log n}\left(\begin{array}{c}
|C| \\
i
\end{array}\right) & \leq\left(\begin{array}{c}
M^{\prime} \log n+\alpha_{0} \log n \\
\alpha_{0} \log n
\end{array}\right) \\
& \leq\left(\frac{\left(M^{\prime}+\alpha_{0}\right) e}{\alpha_{0}}\right)^{\alpha_{0} \log n} \\
& =n^{\alpha_{0} \log \left(\frac{M^{\prime}+\alpha_{0}}{\alpha_{0}} e\right)} \\
& =\sqrt{n}
\end{aligned}
$$

where we have used that $\left(\begin{array}{l}a \\ b\end{array}\right) \leq\left(\frac{a e}{b}\right)^{b}$ for the second inequality and the fact that $\alpha_{0}$ is a root of (6) for the last one.

Since $|V(G) \backslash U| \geq n / 2$ there must be at least two vertices $v_{1}, v_{2} \in V(G) \backslash U$ such that $N_{G}\left[v_{1}\right] \cap C=N_{G}\left[v_{2}\right] \cap C$, and thus $C$ cannot be an identifying code, a contradiction.

The following lemma relates the identifying code number of a graph $G$ to the one of its complement $\bar{G}$.

Lemma 9. Let $G$ be a twin-free graph. If $\bar{G}$ is twin-free, then

$$
\gamma^{I D}(\bar{G}) \leq 2 \gamma^{I D}(G)
$$

Proof. Let $C_{0}$ be a minimum identifying code of $G$. We will show that there exists a set $C_{1}$ of size at most $\gamma^{\mathrm{ID}}(G)-1$ and a special vertex $v$, such that $C=C_{0} \cup C_{1} \cup\{v\}$ is an identifying code of $\bar{G}$.

For the sake of simplicity, we define the following relation. Two vertices $u, v \in V(G)$ are in relation with each other if and only if $N_{G}(u) \cap C_{0}=N_{G}(v) \cap C_{0}$ and $u \nsim v$ (i.e. considering $C_{0}$ in $G, u, v$ are separated by one of $u, v)$. This will be denoted as $u \equiv_{G} v$. It can be checked that this relation is an equivalence relation.

Claim. Every pair of distinct vertices $u \not_{G} v$ is separated by $C_{0}$ in $\bar{G}$.

Proof. By the definition of $\equiv_{G}$, either $N_{G}(u) \cap C_{0} \neq N_{G}(v) \cap C_{0}$ or $u \sim v$.

If $N_{G}(u) \cap C_{0} \neq N_{G}(v) \cap C_{0}$, there exists $w \in C_{0}$ (and $\left.w \notin\{u, v\}\right)$ such that $w \in N_{G}(u) \oplus N_{G}(v)$. Then, $w \in N_{\bar{G}}(u) \oplus N_{\bar{G}}(v)$, hence $w$ still separates $u, v$ in $\bar{G}$.

If $N_{G}(u) \cap C_{0}=N_{G}(v) \cap C_{0}$, then $u \sim v$. If at least one of them belongs to $C_{0}$, then this vertex separates $u, v$ in $\bar{G}$. Otherwise, $u, v \notin C_{0}$ and we have $N_{G}(u) \cap C_{0}=N_{G}[u] \cap C_{0}$ and $N_{G}[v] \cap C_{0}=N_{G}(v) \cap C_{0}$. Hence $N_{G}[u] \cap C_{0}=N_{G}[v] \cap C_{0}$. But then $C_{0}$ does not separate $u, v$ in $G$, a contradiction. 
In particular, this implies that any vertex in an equivalence class of size one is separated by $C_{0}$ from all other vertices in $\bar{G}$.

Claim. If $u \equiv_{G} v$ and both $u, v \notin C_{0}$, then $u=v$.

Proof. Since $u, v \notin C_{0}, N_{G}[u] \cap C_{0}=N_{G}(u) \cap C_{0}$ and $N_{G}[v] \cap C_{0}=N_{G}(v) \cap C_{0}$. Using that they are equivalent, we have that $N_{G}[u] \cap C_{0}=N_{G}[v] \cap C_{0}$. Since $C_{0}$ is an identifying code of $G$, we must have $u=v$.

Claim. Let $U=\left\{u_{1}, \ldots, u_{s}\right\}$ be an equivalence class of $\equiv_{G}$. Then all the pairs in $U$ can be separated in $\bar{G}$ by using $s-1$ vertices.

Proof. We will prove the claim by induction. For $s=2$ it is clearly true: since $\bar{G}$ is twin-free, we can select $w \in N_{\bar{G}}\left[u_{1}\right] \oplus N_{\bar{G}}\left[u_{2}\right]$, and $w$ separates $u$ and $v$ in $\bar{G}$.

For any $s>2$, consider the vertices $u_{1}, u_{2} \in U$ and let $w \in N_{\bar{G}}\left[u_{1}\right] \oplus N_{\bar{G}}\left[u_{2}\right]$. Since $U$ forms a clique in $\bar{G}$, $w \notin U$. Then $w$ splits the set $U$ into $U_{1}$, the set of vertices of $U$ adjacent to $w$ in $\bar{G}$, and $U_{2}$, the set of vertices in $U$ non-adjacent to $w$ in $\bar{G}$. Let $\left|U_{1}\right|=s_{1}$ and $\left|U_{2}\right|=s_{2}$; by construction, $s_{1}, s_{2}<s$.

Now, the pairs of vertices of $U$ with one vertex from $U_{1}$ and one vertex from $U_{2}$ are separated by $w$. By induction, the pairs of vertices in $U_{1}$ can be separated using $s_{1}-1$ vertices and the ones in $U_{2}$ using $s_{2}-1$. Thus we need at most $\left(s_{1}-1\right)+\left(s_{2}-1\right)+1=s-1$ vertices to separate all the pairs of vertices in $U$.

From the previous claims, it is straightforward to deduce that there is a set $C_{1}$ of size at most $\left|C_{0}\right|-1$ vertices that separates all the pairs in $\bar{G}$ that are not separated by $C_{0}$.

Eventually, there might be a unique vertex $v$ such that $N_{\bar{G}}[v] \cap\left(C_{0} \cup C_{1}\right)=\emptyset$ (if there were two such vertices, they would not be separated by $C_{0} \cup C_{1}$, a contradiction). Hence, $C=C_{0} \cup C_{1} \cup\{v\}$ is an identifying code of $\bar{G}$ of size at most $2\left|C_{0}\right|=2 \gamma^{\mathrm{ID}}(G)$.

Proposition 10. For any $M \geq 0$, there exists a constant $c>0$ such that for any set of edges $F \subset E\left(K_{n}\right)$ satisfying $\gamma^{I D}\left(K_{n} \backslash F\right) \leq M \log n,|F| \geq c n \log n$.

Proof. Set $M^{\prime}=M / 2$ and let $c=c_{0}$ be the constant given by Lemma 8 for this $M^{\prime}$. Suppose that there exists a set $F$ of edges, $|F|<c n \log n$ such that $G=K_{n} \backslash F$ satisfies $\gamma^{\mathrm{ID}}(G) \leq M \log n$. By Lemma 9, the graph $\bar{G}$ admits an identifying code of size at most $2 M \log n=M^{\prime} \log n$. By Lemma 8 , we get a contradiction.

Using the former proposition, for any $\delta$ we can provide an example of a graph with minimum degree $\delta$ for which the result of Theorem 3 is asymptotically tight when assuming that $\Delta=\operatorname{Poly}(\delta)$.

For any $\delta>0$, consider the graph $H_{\delta}$ to be the disjoint union of cliques of order $\delta+1$. We may assume that $\delta+1$ divides $n$ for the sake of simplicity. Denote by $H_{\delta}^{(1)}, \ldots, H_{\delta}^{(s)}, s=\frac{n}{\delta+1}$, the cliques composing $H_{\delta}$.

Since $H_{\delta}^{(i)}$ is a connected component, an asymptotically optimal identifying code for $H_{\delta}$ must also be asymptotically optimal for each $H_{\delta}^{(i)}$. By Proposition 10, we must delete at least $\Omega(\delta \log \delta)$ edges from $H_{\delta}^{(i)}$ to get an identifying code of size $O(\log \delta)$.

Thus, one must delete at least $\Omega(s \delta \log \delta)=\Omega(n \log \delta)$ edges from $H_{\delta}$ to get an optimal identifying code.

Corollary 11. For any $\delta=\omega(1)$ and any $M \geq 0$, there exists a constant $c>0$ such that for any set of edges $F \subset E\left(H_{\delta}\right)$ satisfying $\gamma^{I D}\left(H_{\delta} \backslash F\right) \leq M \frac{n \log \delta}{\delta}$, we have $|F| \geq c n \log \delta$. 
We remark that a connected counterexample can also be constructed from $H_{\delta}$ by connecting its cliques using few edges, without affecting the above result.

Corollary 11 implies that Theorem 3 is asymptotically tight when $\Delta=\operatorname{Poly}(\delta)$, since in that case $\log \Delta=$ $O(\log \delta)$. However, when $\delta$ is sub-polynomial with respect to $\Delta$, we do not know if Theorem 3 is asymptotically tight.

\subsection{On the hypothesis}

We conclude this section by discussing the necessity of the hypothesis $\Delta=\omega(1)$ and $\delta \geq 66 \log \Delta$ in Theorem 3 . First note that, if $\Delta$ is bounded by a constant, we need at least $\frac{n}{\Delta+1}=\Theta(n)$ vertices to dominate $G$. Thus, no code of size smaller than $\Theta(n)$ can be obtained by deleting edges of the graph.

On the other hand, the condition $\delta \geq 66 \log \Delta$ in Theorem 3 is also necessary (up to a constant factor) as can be deduced from the following proposition.

Proposition 12. For arbitrarily large values of $\Delta$, there exists a graph $G$ with maximum degree $\Delta$ and minimum degree $\delta=\frac{\log _{2} \Delta}{2}$ such that, for any spanning subgraph $H \subseteq G$,

$$
\gamma^{I D}(H)=(1-o(1)) n
$$

Proof. Consider the bipartite complete graph $G=K_{r, s}$ where $s=2^{2 r}$. Denote by $V_{1}$ the stable set of size $r$ and by $V_{2}$ the stable set of size $s$. Observe that $\delta=r=\frac{\log _{2} s}{2}=\frac{\log _{2} \Delta}{2}$.

For any given twin-free spanning subgraph $H \subseteq G$, let $C \subseteq V(G)$ be an identifying code of $H$. Let us show that most of the vertices in $V_{2}$ must be in $C$. Let $S \subseteq V_{2}$ be the subset of vertices in $V_{2}$ that are not in the code. Thus, for any $u \in S, N_{C}[u]=N_{C}(u)$. Observe that $N_{C}(u) \subseteq V_{1}$, and hence, there are at most $2^{r}$ possible candidates for such $N_{C}(u)$. Since $C$ is dominating and separating all the pairs in $S$, all the subsets $N_{C}(u)$ must be non empty and different, which implies, $|S|<2^{r}$. Hence, we have

$$
|C| \geq\left|V_{2} \backslash S\right| \geq 2^{2 r}-2^{r}=(1-o(1)) 2^{2 r}=(1-o(1)) n .
$$

\section{Consequences of our results}

We now describe consequences of our results on the case when we want to add edges to a graph to decrease its identifying code number, and to the notion of watching systems.

\subsection{Adding edges}

In the previous sections, we have studied how much can the identifying code number decrease when we delete few edges from the original graph. In this section, we discuss the symmetric question of how much can the addition of edges help to decrease this parameter.

The question of how much can a parameter decrease when deleting/adding edges has been already studied for some monotone parameters. However, if the parameter is monotone, only one of either deleting or adding, can 
help to decrease it. One of the interesting facts of studying the identifying code number is that, since it is a non-monotone parameter, we can have similar results for both procedures.

As before, let $G$ be a graph with maximum degree $\Delta$ and minimum degree $\delta$. We aim to find a set of edges $F$ with $F \cap E(G)=\emptyset$ such that $\gamma^{\mathrm{ID}}(G \cup F)$ is small. This set $F$ will be provided by applying Theorem 3 to the graph $\bar{G}$, that has maximum degree $\Delta(\bar{G})=n-1-\delta$ and minimum degree $\delta(\bar{G})=n-1-\Delta$. Thus, it will have size

$$
|F|=O(n \log \Delta(\bar{G}))
$$

and

$$
\gamma^{\mathrm{ID}}(\bar{G} \backslash F)=O\left(\frac{n \log \Delta(\bar{G})}{\delta(\bar{G})}\right)
$$

Since $\bar{G} \backslash F=\overline{G \cup F}$, we have the following corollary of Theorem 3 and Lemma 9

Corollary 13. For any graph $G$ on $n$ vertices with minimum degree $\delta=n-\omega(1)$ and maximum degree $\Delta$ such that $n-\Delta \geq 66 \log (n-\delta)$, there exists a set of edges $F$ with $F \cap E(G)=\emptyset$ of size

$$
|F|=O(n \log (n-\delta)),
$$

such that

$$
\gamma^{I D}(G \cup F)=O\left(\frac{n \log n}{n-\Delta}\right)
$$

This result is also asymptotically tight. Otherwise, by using again Lemma 9, we could translate our case to the case of deleting edges and we would get a contradiction with the optimality of Theorem 3 .

\subsection{Watching systems}

The result of Theorem 3 has a direct application for watching systems, which are a generalization of identifying codes 3 , 4. In a watching system, we can place on each vertex $v$ a set of watchers. To each watcher $w$ placed on $v$, we assign a nonempty subset $Z(w) \subseteq N[v]$, its watching zone. We now ask each vertex to belong to a unique and nonempty set of watching zones; the minimum number of watchers that need to be placed on the vertices of $G$ to obtain a watching system is the watching number $w(G)$ of $G$.

It is clear from the definition that $\gamma(G) \leq w(G) \leq \gamma^{\mathrm{ID}}(G)$, since the vertices of any identifying code form a watching system (where the watching zones are the closed neighborhoods). In fact, even the following holds:

Observation 14. For any twin-free graph $G, w(G) \leq \min \left\{\gamma^{I D}(H)\right.$, where $H$ is a spanning subgraph of $\left.G\right\}$. Indeed, consider the spanning subgraph $H_{0}$ of $G$ with smallest identifying code number, and define the watching system to be the vertices of an optimal identifying code of $H_{0}$, with the watching zones being the closed neighborhoods in $H_{0}$.

In [3, Theorems 2 and 3], the authors propose the following upper bound for graphs with given maximum degree:

Theorem 15 (3]). Let $G$ be a graph with maximum degree $\Delta$, then

$$
\left\lceil\log _{2}(n+1)\right\rceil \leq w(G) \leq \gamma(G)\left\lceil\log _{2}(\Delta+2)\right\rceil .
$$

Note that for any values of parameters $\gamma$ and $\Delta$, the upper bound from the above theorem is tight for the graph consisting of $\gamma$ disjoint copies of a star on $\Delta+1$ vertices. 
It is well-known (see e.g. 2, Theorem 1.2.2]) that the domination number of a graph with minimum degree $\delta$ satisfies

$$
\gamma(G) \leq(1+o(1)) \frac{n \log \delta}{\delta} .
$$

This bound is sharp and, in particular, the "typical" $\delta$-regular graph is an asymptotically tight example. Indeed, for such a "typical" graph $G$, the upper bound of Theorem 15 gives

$$
w(G) \leq \gamma(G)\lceil\log \Delta+2\rceil=\Omega\left(\frac{n \log ^{2} \delta}{\delta}\right) .
$$

By Observation 14, a direct corollary of Theorem 3 is the following:

Corollary 16. For any graph $G$ on $n$ vertices with minimum degree $\delta \geq 66 \log \Delta$ and maximum degree $\Delta=\omega(1)$, we have:

$$
w(G) \leq 134 \frac{n \log \Delta}{\delta} .
$$

Note that this bound improves Theorem 15 when the maximum degree is $\Delta=\operatorname{Poly}(\delta)$.

\section{Concluding remarks and open questions}

1. The kind of results we provide in this paper can be connected to the notion of resilience. Given a graph property $\mathcal{P}$, the global resilience of $G$ with respect to $\mathcal{P}$ is the minimum number of edges one has to delete to obtain a graph not satisfying $\mathcal{P}$. The resilience of monotone properties is well studied, in particular, in the context of random graphs 27 .

Our result can be interpreted in terms of the resilience of the following (non-monotone) property $\mathcal{P}$ : " $G$ has a large identifying code number in terms of its degree parameters, $\delta$ and $\Delta$ ". For any graph $G$ satisfying the hypothesis $\Delta=\omega(1)$ and $\delta \geq 66 \log \Delta$, Theorem 3 can be stated as: the resilience of $G$ with respect to $\mathcal{P}$ is $O(n \log \Delta)$. Moreover, Corollary [1] shows that there are graphs that attain this value of the resilience.

2. In Theorem 3, we show the existence of a small identifying code for a large spanning subgraph of $G$. However, our proof is not constructive and, besides, the probability that such pair exists is exponentially small, due to the use of the local lemma. The algorithmic version of the local lemma proposed by Moser and Tardos, allows to explicitly find a configuration that avoids all the bad events $E_{u v}$, when these events are determined by a finite set of mutually independent random variables. Unfortunately, this is not the case here, since $E_{u v}$ depends on the random variables determining the existence of certain edges close to $u v$. These random variables are not independent because of the definition of $p_{u v}$.

On the other hand, if we do not want to argue in terms of the maximum degree $\Delta$, one can show that by deleting a set of $O(n \log n)$ random edges we have an identifying code of size $O\left(\frac{n \log n}{\delta}\right)$ with probability $1-o(1)$. In such a case, the proof provides a randomized algorithm which constructs the desired code for almost all subgraphs.

3. Note that a notion similar to identifying codes, locating-dominating sets, was also extensively studied in the literature (see e.g. 24] for many references). A set $C$ of vertices of $G$ is a locating-dominating set if $C$ is a dominating set which separates all pairs of vertices in $V(G) \backslash C$. It follows that any identifying code is a locating-dominating set, hence Theorem 3 also holds for this notion. In fact, the proof of Corollary 11 can be adapted for this case too. 
4. As further research, it would be very interesting to close the gap between the result in Theorem 3 and the lower bound given by the example in Corollary 11 Motivated by this example, we ask the following question:

Question 17. Is it true that for any graph $G$ with minimum degree $\delta$, there exists a subset of edges $F \subset E(G)$ of size

$$
|F|=O(n \log \delta),
$$

such that

$$
\gamma^{I D}(G \backslash F)=O\left(\frac{n \log \delta}{\delta}\right) ?
$$

It seems to us that the techniques used in this paper will not provide an answer to the previous question. The main obstacle is the use of the local lemma, which forces us to take into account the role of the maximum degree of $G$.

\section{References}

[1] N. Alon. A note on network reliability. Discrete probability and algorithms (Minneapolis, MN, 1993), IMA Volumes in Mathematics and its Applications 72:11-14, 1995.

[2] N. Alon and J. H. Spencer. The probabilistic method, 3rd edition, Wiley-Interscience, 2008.

[3] D. Auger, I. Charon, O. Hudry and A. Lobstein. Watching systems in graphs: an extension of identifying codes. To appear in Discrete Applied Mathematics.

[4] D. Auger, I. Charon, O. Hudry and A. Lobstein. Maximum size of a minimum watching system and the graphs achieving the bound. To appear in Discrete Applied Mathematics.

[5] L. Babai. On the complexity of canonical labeling of strongly regular graphs. SIAM Journal of Computing $9(1): 212-216,1980$.

[6] N. Bertrand. Codes identifiants et codes localisateurs-dominateurs sur certains graphes. Master thesis, ENST, France, June 2001.

[7] J. A. Bondy. Induced subsets. Journal of Combinatorial Theory, Series B 12(2):201-202, 1972.

[8] I. Charon, I. Honkala, O. Hudry and A. Lobstein. Minimum sizes of identifying codes in graphs differing by one vertex. Cryptography and Communications 5(2):1-18, 2013.

[9] I. Charon, O. Hudry and A. Lobstein. Extremal cardinalities for identifying and locating-dominating codes in graphs. Discrete Mathematics 307(3-5):356-366, 2007.

[10] F. Chung and P. Horn. The spectral gap of a random subgraph of a graph. Internet Mathematics 4(23):225-244, 2007.

[11] K. M. J. De Bontridder, B. V. Halldórsson, M. M. Halldórsson, C. A. J. Hurkens, J. K. Lenstra, R. Ravi and L. Stougie. Approximation algorithms for the test cover problem. Mathematical Programming Series B 98:477-491, 2003.

[12] F. Foucaud, E. Guerrini, M. Kovše, R. Naserasr, A. Parreau and P. Valicov. Extremal graphs for the identifying code problem. European Journal of Combinatorics 32(4):628-638, 2011. 
[13] F. Foucaud, R. Klasing, A. Kosowski and A. Raspaud. On the size of identifying codes in triangle-free graphs. Discrete Applied Mathematics 160(10-11):1532-1546, 2012.

[14] F. Foucaud and G. Perarnau. Bounds for identifying codes in terms of degree parameters. The Electronic Journal of Combinatorics 19:P32, 2012.

[15] A. Frieze and M. Krivelevich. On the non-planarity of a random subgraph. ArXiv e-prints, 2012.

[16] A. Frieze, R. Martin, J. Moncel, M. Ruszinkó and C. Smyth. Codes identifying sets of vertices in random networks. Discrete Mathematics 307(9-10):1094-1107, 2007.

[17] S. Gravier and J. Moncel. On graphs having a $V \backslash\{x\}$ set as an identifying code. Discrete Mathematics 307(3-5):432-434, 2007.

[18] T. W. Haynes, D. J. Knisley, E. Seier and Y. Zou. A quantitative analysis of secondary RNA structure using domination based parameters on trees. BMC Bioinformatics 7:108, 2006.

[19] M. G. Karpovsky, K. Chakrabarty, and L. B. Levitin. On a new class of codes for identifying vertices in graphs. IEEE Transactions on Information Theory, 44:599-611, 1998.

[20] J. H. Kim, O. Pikhurko, J. Spencer and O. Verbitsky. How complex are random graphs in First Order logic? Random Structures and Algorithms 26(1-2):119-145, 2005.

[21] M. Krivelevich, C. Lee and B. Sudakov. Long paths and cycles in random subgraphs of graphs with large minimum degree. ArXiv e-prints, 2012.

[22] M. Krivelevich and B. Sudakov. The phase transition in random graphs - a simple proof. ArXiv e-prints, 2012.

[23] M. Laifenfeld, A. Trachtenberg, R. Cohen and D. Starobinski. Joint monitoring and routing in wireless sensor networks using robust identifying codes. Proc. IEEE Broadnets 200\%, pp. 197-206, 2007.

[24] A. Lobstein. Watching systems, identifying, locating-dominating and discriminating codes in graphs: a bibliography. http://www.infres.enst.fr/ lobstein/debutBIBidetlocdom.pdf

[25] J. Moncel. On graphs on $n$ vertices having an identifying code of cardinality $\log _{2}(n+1)$. Discrete Applied Mathematics, 154(14):2032-2039, 2006.

[26] B. M. E. Moret and H. D. Shapiro. On minimizing a set of tests. SIAM Journal of Scientifical and Statistical Computation 6(4):983-1003, 1985.

[27] B. Sudakov and and V. H. Vu. Local resilience of graphs, Random Structures Algorithms 33(4):409-433, 2008.

[28] R. Ungrangsi, A. Trachtenberg and D. Starobinski. An implementation of indoor location detection systems based on identifying codes. Proc. Intelligence in Communication Systems, INTELLCOMM 2004, Lecture Notes in Computer Science 3283:175-189, 2004. 